\title{
Electromyographic Manifestations of Fatigue Correlate With Pulmonary Function, 6-Minute Walk Test, and Time to Exhaustion in COPD
}

\author{
Gennaro Boccia PhD, Davide Dardanello PT, Nicoletta Rinaldo PhD, Giuseppe Coratella PhD, \\ Federico Schena MD PhD, and Alberto Rainoldi PhD
}

\begin{abstract}
BACKGROUND: The aim of this study was to investigate whether electromyographic manifestations of fatigue and exercise tolerance were related to stage of disease in men with a COPD diagnosis. METHODS: Fourteen men with COPD with a diagnosis of mild to severe air flow obstruction were involved in 2 separate testing sessions. The first one consisted of a pulmonary function $\left(\mathrm{FEV}_{1}\right.$ and $\left.\mathrm{FEV}_{1} / \mathrm{FVC}\right)$ and an exercise tolerance assessment using the 6-min walk test. During the second session, a multichannel surface electromyography was recorded from vastus medialis and vastus lateralis muscles during an isometric knee extension at $70 \%$ of maximum voluntary contraction. The slope of muscle fiber conduction velocity during the contraction was calculated as the index of fatigue. RESULTS: Conduction velocity slope significantly correlated with $\mathrm{FEV}_{1}$ (vastus medialis: $\mathrm{r}=0.86, P<.001$; vastus lateralis: $\mathrm{r}=0.68, P=.01$ ), $\mathrm{FEV}_{1} / \mathrm{FVC}$ (vastus medialis: $\mathrm{r}=0.70, P=.006$ ), and 6 -min walk test (vastus medialis: $\mathrm{r}=0.72, P=.005$; vastus lateralis: $r=0.80, P=.001)$. CONCLUSIONS: The electromyographic manifestations of fatigue during sustained quadriceps contraction significantly correlated with disease severity and exercise tolerance in moderate to severe COPD. Key words: COPD; neuromuscular fatigue; electromyography; 6-minute walk test; muscle endurance; vastus medialis; vastus lateralis. [Respir Care 2015;60(9):1295-1302. (C) 2015 Daedalus Enterprises]
\end{abstract}

\section{Introduction}

COPD is the fourth-leading cause of death worldwide, and it represents an important public health challenge. ${ }^{1}$ It is characterized by progressive air flow limitation, not fully reversible, due to an abnormal inflammatory response of the lungs. ${ }^{2}$ COPD patients develop a great number of extrapulmonary manifestations, such as lower limb muscle wasting, redistribution of fiber type ratio toward type II fibers, ${ }^{3}$ and reduction in muscle oxidative capacity. ${ }^{4}$ These

Drs Boccia, Dardanello, and Rainoldi are affiliated with the Motor Science Research Center, School of Exercise and Sport Sciences, SUISM, Department of Medical Sciences, University of Turin, 12, Piazza Bernini, Torino, Italy. Drs Rinaldo, Coratella, and Schena are affiliated with the Department of Neurological and Movement Sciences, University of Verona, Via Casorati 43, Verona, Italy. Drs Boccia and Schena are also affiliated with the CeRiSM Research Center "Sport, Mountain, and Health," Via del Ben 5/b, Rovereto, Italy.

This work was supported by the grant "Project HExEC," PQR FESR 2007/2013, funding for innovation projects. The authors have disclosed no conflicts of interest. comorbidities seem to be the main exercise-limiting factors in COPD patients ${ }^{5}$ with a significant reduction in strength and endurance performances. ${ }^{4}$ Recent studies have shown that lower limb muscle strength and fatigability are strictly connected with daily physical activity. In addition, they predict mortality, morbidity, and patient quality of life better than lung function measures. ${ }^{6}$

Fatigability is usually assessed by the time to exhaustion in conjunction with electromyography (EMG). Surface EMG has been used to characterize neuromuscular fatigue during isometric tasks. ${ }^{7}$ We refer to myoelectric manifestations of fatigue as all of the changes in surface EMG variables occurring during sustained muscle contractions. ${ }^{8}$ Myoelectric manifestations of fatigue relate to

\footnotetext{
Correspondence: Alberto Rainoldi PhD, Motor Science Research Center, School of Exercise and Sport Sciences, SUISM, Department of Medical Sciences, University of Turin, 12 piazza Bernini, 10143 Torino, Italy. E-mail: alberto.rainoldi@unito.it.
}

DOI: $10.4187 /$ respcare. 04138 
alteration of muscle metabolic conditions, in particular to decrease of muscle fiber $\mathrm{pH} .{ }^{9}$ Multichannel surface EMG provides the possibility of estimating the propagation velocity of motor unit action potential along the sarcolemma. ${ }^{10}$ Muscle fiber conduction velocity is an important physiological variable related to the size of recruited fibers. ${ }^{11,12}$ During submaximal isometric contraction, EMG variables show typical trends: decrement of conduction velocity, decrement of mean power spectral frequency, and increment of average rectified value. ${ }^{13}$ Greater recruitment of type II muscle fibers has been demonstrated to generate greater myoelectric manifestations of fatigue, measured as a larger rate of changes of EMG variables over time. ${ }^{14,15}$ Therefore, multichannel surface EMG technology has been proposed as a tool for the noninvasive characterization of muscle phenotype composition. ${ }^{16}$ Moreover, conduction velocity has been demonstrated to be a more reliable and sensitive index of fatigue with respect to power spectral frequency. ${ }^{12}$

Monitoring EMG changes can provide useful insight to characterize fatigability in COPD, in addition to the widely used outcome measures of fatigue, such as mechanical task failure and decreased force capability after sustained or intermittent contractions. ${ }^{17}$ Since myoelectric manifestations of fatigue are related to muscle fiber composition and metabolic conditions, they provide indirect but useful information on important peripheral muscle abnormalities of patients with COPD. Moreover, EMG changes occur starting at the beginning of a sustained contraction; consequently, they can be quantified before the task failure without requiring exhausting effort. ${ }^{18}$ Hence, it is reasonable to hypothesize that monitoring EMG changes can characterize fatigability in COPD, avoiding the discomfort of exhausting effort. For these reasons, the first aim of the study was to assess the indexes of EMG fatigue in men with a COPD diagnosis, during submaximal isometric contractions of quadriceps muscle, to investigate whether they were related to stage of disease and exercise tolerance. The second aim of the study was to re-estimate the same indexes using only the first $10 \mathrm{~s}$ of the contraction to verify whether they still correlated with the stage of disease and exercise tolerance well before the task failure.

\section{Methods}

\section{Participants}

Fourteen men with a COPD diagnosis of mild to very severe air flow obstruction were recruited for the study. All participants were clinically stable, without any exacerbation of their disease in the 4 weeks before the study. The exclusion criteria were: presence of any respiratory tract infections, other respiratory diagnosis, major comorbidity (eg, cardiovascular diseases, diabetes, or cancer),

\section{QUICK LOOK}

\section{Current knowledge}

COPD is the fourth-leading cause of death worldwide and an important public health challenge. COPD is characterized by irreversible progressive air flow limitation, due to an abnormal inflammatory response. COPD subjects develop lower limb muscle wasting and reduction in muscle oxidative capacity, resulting in exercise limitation. Lower limb muscle strength and fatigability are connected with daily physical activity and predict mortality, morbidity, and patient quality of life better than lung function measures.

\section{What this paper contributes to our knowledge}

The rate of change of conduction velocity during sustained quadriceps contraction significantly correlated with disease severity and exercise tolerance in mild to very severe COPD. The rate of change of conduction velocity calculated in the first $10 \mathrm{~s}$ of contraction was found to correlate with the time to exhaustion, disease severity, and exercise tolerance. The electromyographic manifestations of fatigue during sustained quadriceps contraction significantly correlated with disease severity and exercise tolerance in moderate to severe COPD.

and lower limb joint diseases. All participants provided their written informed consent before participation in the experiments. The research was previously approved by the Ethics Committee of the Department of Neurological and Movement Science, University of Verona, in accordance with the Declaration of Helsinki and the ethical standards of the International Journal of Sports Medicine. ${ }^{19}$

\section{Procedure}

This investigation is an experimental, cross-sectional study. Participants were involved in 2 separate testing sessions. The first one consisted of pulmonary function evaluation and subsequently exercise tolerance assessment. Three days later, surface EMG signals were recorded from the quadriceps muscle during submaximal isometric knee extensor contractions. Participants were instructed to refrain from performing strenuous physical activity in the $24 \mathrm{~h}$ before the experimental session.

During the first session, $\mathrm{FEV}_{1}$ and $\mathrm{FVC}$ values were measured according to the American Thoracic Society /European Respiratory Society Task Force methods, ${ }^{20}$ using a pulmonary function instrument with computer processing (Quark, Cosmed, Italy). After pulmonary function assessment, exercise tolerance was investigated with 
the 6-min walk test (6MWT), conducted according to the standardized protocol. ${ }^{21}$ Subjects were instructed to walk down a $30-\mathrm{m}$ shuttle hallway at their own pace while attempting to cover as much ground as possible in the allotted $6 \mathrm{~min}$.

During the second session, knee extensor isometric peak torque was measured at $60^{\circ}$ of knee flexion $\left(0^{\circ}=\right.$ full extension) using an isokinetic device (Cybex NORM dynamometer, Ronconcoma, New York). Participants were seated upright on the dynamometer chair and secured using a seatbelt. Participants became familiarized with the device by performing 10 submaximal isometric contractions. Then 2 maximal voluntary contractions $5 \mathrm{~s}$ long were exercised at $60^{\circ}$, separated by $5 \mathrm{~min}$ of rest. If maximal voluntary contractions differed $>5 \%$ from each other, a third one was performed. During maximal voluntary contraction, participants received visual feedback of exerted torque and strong verbal encouragement to reach their highest level in each trial. The highest maximal voluntary contraction was used to calculate submaximal loads.

Five min after the last maximal voluntary contraction, participants were involved in fatiguing submaximal position tasks. They were instructed to maintain a constant knee extension at $60^{\circ}$ until voluntary exhaustion, supporting a load equivalent to $70 \%$ of the torque exerted during the maximal voluntary contraction. Participants received on a display visual feedback about the actual knee angle and were instructed to hold the target value of $60^{\circ}$. The task was considered failed when the limb moved $\pm 5^{\circ}$ for $>3 \mathrm{~s}$. The time to exhaustion was calculated as the index of fatigability. Standardized encouragement was provided by an operator to maintain the limb as stable as possible.

\section{EMG Recording}

Myoelectric signals were recorded from vastus medialis and vastus lateralis muscles of the dominant leg in single differential configuration using linear adhesive electrode arrays of 8 electrodes with 5-mm interelectrode distance (OT Bioelettronica, Torino, Italy). Before the placement of the electrode arrays, the skin was slightly abraded with paste and cleaned with water in accordance with SENIAM (surface EMG for the non-invasive assessment of muscles) recommendations for skin preparation. ${ }^{22}$ The optimal position and orientation of the array was sought for each muscle on the basis of visual inspection of the surface EMG signals. The sites with clear propagation of muscle fiber action potential and the main innervation zones were identified using a dry linear array of 16 electrodes with a 5-mm interelectrode distance (OT Bioelettronica). The adhesive electrode arrays were then placed parallel to muscle fibers, distally with respect to the innervation zone, where unidirectional propagation of the motor unit action poten- tials was detected. To assure proper electrode-skin contact, electrode cavities of the arrays were filled with 20-30 $\mu$ l of conductive paste (Spes-Medica, Battipaglia, Italy). The electrode arrays were fixed using extensible dressing (Fixomull, Beiersdorf, Hamburg, Germany). The surface EMG signals were amplified, sampled at $2,048 \mathrm{~Hz}$, band-passfiltered (3-decibel bandwidth, 20-450 Hz, 12 decibels/ octave slope on each side), and converted to digital data by a 12-bit analog-to-digital converter (EMGUSB, 64-channel amplifier; OT Bioelettronica). Samples were visualized during acquisition and then stored in a personal computer using OT BioLab 1.8 software (OT Bioelettronica) for further analysis.

\section{Data Analysis}

The EMG signals were visually inspected to select the best channels to use for EMG variable estimations. Mean power spectral frequency, average rectified value, and conduction velocity of EMG signals were computed off-line with numerical algorithms ${ }^{10}$ using non-overlapping signal epochs of $0.5 \mathrm{~s}$. The correlation coefficient between the 2 adjacent double differential signals was calculated, and if the correlation coefficient was $<80 \%$, the recorded signals were excluded from the analysis. Conduction velocities were calculated among all of the accepted channels. Average rectified value and mean power spectral frequency estimates were averaged among all of the accepted channels.

Linear regression was applied to the time course of EMG data to calculate the initial value, rate of change, and normalized rate of change (calculated as the percentage ratio between rate of change and initial value) of mean power spectral frequency and conduction velocity. ${ }^{9}$ Normalized rate of change estimates were used as indexes of myoelectric fatigue. To test whether indexes of myoelectric fatigue can be suitable in the case of short non-exhausting contraction, linear regression of EMG estimates were calculated for 2 lengths of analysis: (1) the complete time course of contraction until task failure and (2) the first $10 \mathrm{~s}$ of contraction (20 epochs of $0.5 \mathrm{~s}$ ) (see Table 2). The cut-off of $10 \mathrm{~s}$ was chosen because it was expected to be well before the task failure of all participants.

\section{Statistical Analysis}

The normal distribution of data was verified with the Kolmogorov-Smirnov test. Age, body mass index, lung function, distance covered in 6MWT, time to task failure, and myoelectric indexes of fatigue were expressed as mean $\pm \mathrm{SD}$, by presenting a parametric distribution. Pearson correlations were used to test whether myoelectric indexes of fatigue were related to pulmonary function, 6MWT, and time to exhaustion. Two-way analyses 
Table 1. Participant Characteristics and Performances (Mean \pm SD) for the Whole Study Population and for Each Disease Stage

\begin{tabular}{lcccccccc}
\hline \hline & $n$ & Age, $\mathrm{y}$ & $\mathrm{BMI}, \mathrm{kg} / \mathrm{m}^{2}$ & $\mathrm{FEV}_{1}, \%$ of predicted & $\mathrm{FEV}_{1} / \mathrm{FVC}$ & BODE & 6MWD, m & Time to exhaustion, $\mathrm{s}$ \\
\hline COPD & 14 & $67 \pm 5$ & $28.1 \pm 4.2$ & $59.2 \pm 19.2$ & $57.1 \pm 13.6$ & $2.4 \pm 0.7$ & $441 \pm 107$ & $23 \pm 5$ \\
Mild & 2 & $65 \pm 2$ & $30.2 \pm 10.2$ & $81.0 \pm 0.5$ & $65.9 \pm 9.8$ & $1.7 \pm 0.7$ & $507 \pm 31$ & $24 \pm 2$ \\
Moderate & 8 & $67 \pm 5$ & $28.6 \pm 3.7$ & $65.3 \pm 10.1$ & $60.3 \pm 10.1$ & $2.2 \pm 0.4$ & $488 \pm 52$ & $25 \pm 5$ \\
Severe & 3 & $69 \pm 2$ & $25.8 \pm 3.2$ & $42.7 \pm 8.1$ & $50.9 \pm 17.8$ & $3.0 \pm 0.0$ & $365 \pm 75$ & $20 \pm 7$ \\
Very severe & 1 & 59 & 26.0 & 16.0 & 31.9 & 4 & 167 & 18
\end{tabular}

$\overline{6 \mathrm{MWD}=6 \text {-min walk }}$ distance

$\mathrm{BMI}=$ body mass index

BODE = body mass index, airflow obstruction, dyspnea, and exercise
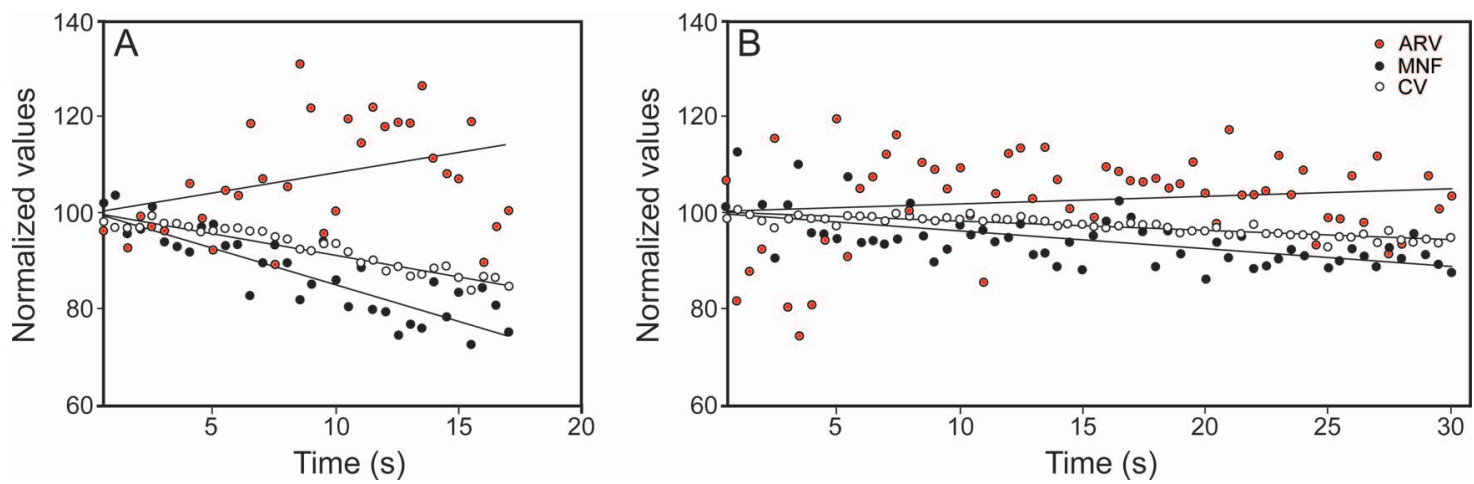

Fig. 1. Representative examples of a fatigue plot diagram of very severe COPD subjects $(A)$ and a mild COPD subject (B). Time courses of average rectified value (ARV), mean power spectral frequency (MNF), and muscle fiber conduction velocity (CV) are represented for each epoch (0.5-s length) of the isometric contraction at $70 \%$ of maximal voluntary contraction. Each EMG variable is normalized with respect to its initial value, and the slope of the regression line represents an index of myoelectric fatigue: the greater the rate of change of surface EMG variables, the greater the manifestations of fatigue. A: Severe subjects showed a shorter time to exhaustion and steeper rate of change of EMG variables as compared with the subject in B (mild COPD).

of variance ( 2 length analyses $\times 2$ muscles) were conducted to detect differences in surface EMG variables (conduction velocity and mean power spectral frequency). When interaction results were significant, the post hoc analysis was performed using a $t$ test with Bonferroni correction. The threshold for statistical significance was set to $P<.05$.

\section{Results}

Participant characteristics and performances are reported in Table 1. The time to task failure during the sustained isometric contraction was $23 \pm 5 \mathrm{~s}$ (range 18-30 s), and then the cut-off of $10 \mathrm{~s}$ to analyze the EMG preceded the task failure of each participant.

\section{EMG Results}

All conduction velocity estimates were found in the physiological range (between 3.5 and $5.7 \mathrm{~m} / \mathrm{s}$ ), and all EMG signals chosen for variable estimates showed a high correlation coefficient $(85 \pm 5 \%)$. Representative examples of fatigue plots for 2 COPD subjects are reported in Figure 1. The fatigue plots represent the time courses of each EMG variable during the sustained isometric contraction at $70 \%$ of maximal voluntary contraction.

Two-way analyses of variance did not show significant interaction for contraction length analysis $\times$ muscles (conduction velocity: $P=.81$; mean power spectral frequency: $P=.37$ ), and no main effects for muscles (conduction velocity: $P=.79$; mean power spectral frequency: $P=.29$ ) and for length analysis (conduction velocity: $P=.66$; mean power spectral frequency: $P=.72$ ) were found. Myoelectric indexes of fatigue are reported in Table 1, and differences between lengths of analysis are shown in Table 2.

The correlation analysis between EMG variables and pulmonary function, 6MWT, and time to task failure is reported in Table 3 (for the complete contraction) and in Table 4 (for the first $10 \mathrm{~s}$ of contraction). In general, the slope of conduction velocity correlated better with pulmonary function and time to task failure than did the slope of mean power spectral frequency (Fig. 2). 
Table 2. Differences Between Lengths of Analysis

\begin{tabular}{lccr}
\hline \hline & Complete & First $10 \mathrm{~s}$ & $P$ \\
\hline CV rate of change, $\% / \mathrm{s}$ & & & \\
VM & $-0.41 \pm 0.38$ & $-0.35 \pm 0.48$ & .39 \\
VL & $-0.41 \pm 0.37$ & $-0.33 \pm 0.47$ & .17 \\
MNF rate of change, $\% / \mathrm{s}$ & & & .33 \\
VM & $-0.59 \pm 0.62$ & $-0.73 \pm 0.71$ & .86 \\
VL & $-0.58 \pm 0.50$ & $-0.60 \pm 0.68$ & \\
& & & \\
\hline Mean \pm SD of electromyography of muscle fiber conduction velocity and mean power & \\
spectrum frequency from vastus lateralis and vastus medialis obliquus are shown. There were \\
no differences between lengths of analysis in all conditions. \\
CV $=$ conduction velocity \\
VM $=$ vastus medialis \\
VL $=$ vastus lateralis \\
MNF $=$ mean power spectrum frequency
\end{tabular}

\section{Discussion}

We investigated quadriceps muscle since alterations in muscle strength and fatigability in COPD are strictly related to quality of life and use of health-care resources. ${ }^{4}$ This study provides further insights into neuromuscular fatigue in COPD, evaluating for the first time muscle fiber conduction velocity during an isometric contraction of knee extensor muscles. The main finding of this study was the significant correlation between rate of change of conduction velocity and both pulmonary function and the 6MWT. Moreover, the correlation between the conduction velocity index of fatigue and pulmonary function was significant (for the vastus medialis) also when the first $10 \mathrm{~s}$ of contraction was analyzed.

The rate of change of conduction velocity correlated with $\mathrm{FEV}_{1}$ and $\mathrm{FEV}_{1} / \mathrm{FVC}$, suggesting that myoelectric fatigability of COPD subjects is associated with pulmonary function and disease severity. This is an important finding since this can offer new representative indexes of fatigability and quadriceps muscle dysfunction in COPD. ${ }^{17}$ A greater proportion of type II muscle fibers has been demonstrated to generate greater myoelectric manifestations of fatigue, measured as a greater decrease of conduction velocity over time. ${ }^{14,15,23}$ Our results are in accordance with the meta-analysis of Gosker et al, ${ }^{3}$ indicating that fiber type proportion was correlated with $\mathrm{FEV}_{1}$ and $\mathrm{FEV}_{1} / \mathrm{FVC}$ in subjects with moderate to severe COPD. Protocols based on conduction velocity estimates, which were similar to the one adopted herein, have been proposed as a potential tool for noninvasive characterization of muscle fiber composition. ${ }^{16}$ Indeed, several previous studies interpreted differences in the rate of change of conduction velocity during continuous isometric contractions as alterations in fiber type composition due to physical training, ${ }^{24}$ pathologies, ${ }^{25-27}$ and aging. ${ }^{28}$ In addition to fiber type shifting toward more glycolytic fibers, other concurrent changes reported in the literature may contribute to increasing EMG fatigue with increasing severity of the disease. ${ }^{4}$ Indeed, capillary density, capillary muscle fiber contact reduction, and lower levels of oxidative enzyme activity are usually related to reductions in muscle oxidative capacity. ${ }^{4}$ The rapid rise in blood lactate levels during exercise is responsible for the decrease in muscle $\mathrm{pH}^{29}$ that is related to the marked conduction velocity reduction with fatigue detected in our COPD group. Data in the literature support the presence of muscle dysfunction even in the early stages of COPD, and the resulting compromise of exercise tolerance also appears in subjects with mild to moderate disease. ${ }^{30}$

The conduction velocity-related indexes of myoelectric fatigue detected during the sustained contraction were found to correlate with the time to task failure (vastus medialis: $r=0.72$; vastus lateralis: $r=0.80$ ) and with the distance covered in 6MWT (vastus medialis: $r=0.77$ ). ${ }^{4}$ It is noteworthy that the 6MWT and the sustained contraction at $70 \%$ of maximal voluntary contraction rely on different energetic supplies, being mainly aerobic in the former and anaerobic in the latter task. Despite this difference, the performance in these 2 tasks might have been limited by the same impairment related to disease severity.

\section{EMG Indexes of Fatigue}

To the best of our knowledge, this is the first study assessing muscle fiber conduction velocity in COPD individuals. When we calculated the rates of change of EMG variables during the complete contraction, conduction velocity, compared with mean power spectral frequency, resulted in better correlation with $\mathrm{FEV}_{1}$. Remarkably, when $10 \mathrm{~s}$ of contraction was used to calculate the index of fatigue, only conduction velocity correlated with $\mathrm{FEV}_{1}$ in vastus medialis, and a trend was shown in vastus lateralis. The findings herein confirmed that when fatiguing protocols are used to assess neuromuscular fatigue, the conduction velocity values provide more affordable and sensitive outcomes than spectral variables. ${ }^{12}$ The poor sensitivity of the mean power spectral frequency rate of change may explain why a previous study ${ }^{31}$ failed to detect differences in the mean power spectral frequency rate of change between COPD subjects and healthy adults.

Moreover, the rate of change of conduction velocity calculated in the first $10 \mathrm{~s}$ significantly correlated with time to task failure. This is an important finding, since it suggests that conduction velocity rate of change can be used to assess fatigability also in non-exhausting tasks. Therefore, monitoring conduction velocity during a sustained contraction can probably serve as an alternative to muscle endurance assessment, avoiding the discomfort of physical exhaustion and the bias induced by motivational factors. 
Table 3. EMG Indexes of Fatigue Based on Complete Contraction

\begin{tabular}{|c|c|c|c|c|c|c|c|c|c|c|c|c|}
\hline & \multicolumn{3}{|c|}{$\mathrm{FEV}_{1}$} & \multicolumn{3}{|c|}{$\mathrm{FEV}_{1} / \mathrm{FVC}$} & \multicolumn{3}{|c|}{$6 \mathrm{MWT}$} & \multicolumn{3}{|c|}{ Time to Exhaustion } \\
\hline & $\mathrm{r}$ & $r^{2}$ & $P$ & $\mathrm{r}$ & $r^{2}$ & $P$ & $\mathrm{r}$ & $r^{2}$ & $P$ & $\mathrm{r}$ & $r^{2}$ & $P$ \\
\hline CV VM & 0.87 & 0.75 & $<.001$ & 0.709 & 0.50 & .006 & 0.77 & 0.60 & .001 & 0.72 & 0.52 & .005 \\
\hline CV VL & 0.68 & 0.47 & .01 & 0.45 & 0.20 & .12 & 0.52 & 0.27 & .067 & 0.80 & 0.64 & .001 \\
\hline MNF VM & 0.57 & 0.33 & .040 & 0.44 & 0.19 & .14 & 0.49 & 0.24 & .09 & 0.63 & 0.39 & .02 \\
\hline MNF VL & 0.50 & 0.25 & .08 & 0.42 & 0.18 & .15 & 0.39 & 0.15 & .19 & 0.64 & 0.41 & .02 \\
\hline
\end{tabular}

Data from the whole contraction duration are shown. Correlation coefficients (Pearson r) between EMG fatigue indexes and pulmonary function, 6-min walk test, and time to exhaustion are shown. $6 \mathrm{MWT}=6$-min walk test

$\mathrm{CV}=$ muscle fiber conduction velocity

$\mathrm{VM}=$ vastus medialis

$\mathrm{VL}=$ vastus lateralis

$\mathrm{MNF}=$ mean power spectrum frequency

Table 4. EMG Indexes of Fatigue Based on the First $10 \mathrm{~s}$ of Contraction

\begin{tabular}{|c|c|c|c|c|c|c|c|c|c|c|c|c|}
\hline & \multicolumn{3}{|c|}{$\mathrm{FEV}_{1}$} & \multicolumn{3}{|c|}{$\mathrm{FEV}_{1} / \mathrm{FVC}$} & \multicolumn{3}{|c|}{$6 \mathrm{MWT}$} & \multicolumn{3}{|c|}{ Time to Exhaustion } \\
\hline & $\mathrm{r}$ & $r^{2}$ & $P$ & $\mathrm{r}$ & $r^{2}$ & $P$ & $\mathrm{r}$ & $r^{2}$ & $P$ & $\mathrm{r}$ & $r^{2}$ & $P$ \\
\hline CV VM & 0.73 & 0.53 & .005 & 0.57 & 0.32 & .043 & 0.57 & 0.32 & .042 & 0.67 & 0.45 & .01 \\
\hline CV VL & 0.49 & 0.24 & .09 & 0.27 & 0.07 & .37 & 0.25 & 0.06 & .41 & 0.75 & 0.56 & .003 \\
\hline MNF VM & 0.02 & 0.00 & .96 & 0.05 & 0.00 & .86 & 0.02 & 0.00 & .96 & 0.53 & 0.28 & .065 \\
\hline MNF VL & 0.07 & 0.00 & .82 & 0.07 & 0.01 & .81 & 0.07 & 0.00 & .83 & 0.55 & 0.30 & .053 \\
\hline
\end{tabular}

$\overline{\text { Data from the first } 10} \mathrm{~s}$ of contraction are shown. Correlation coefficients (Pearson r) between EMG fatigue indexes and pulmonary function, 6-min walk test, and time to exhaustion are shown. $6 \mathrm{MWT}=6$-min walk test

$\mathrm{CV}=$ muscle fiber conduction velocity

$\mathrm{VM}=$ vastus medialis

$\mathrm{VL}=$ vastus lateralis

$\mathrm{MNF}=$ mean power spectrum frequency

\section{Muscle Differences}

We compared 2 portions of quadriceps muscle, vastus lateralis and vastus medialis, which showed the same trends in the myoelectric indexes of fatigue. However, it seemed that fatigue indexes better correlated with lung function and exercise tolerance for vastus medialis than for vastus lateralis. Most evidence regarding COPD alteration at the histochemical level has come from investigations on the vastus lateralis muscle only. ${ }^{3}$ The vastus lateralis is readily accessible for biopsy and has been used often, since it is considered representative of the whole quadriceps muscle femoris, in both aging and COPD studies. Since vastus lateralis is only a part of the whole quadriceps, we decided to expand the knowledge of another portion of that muscular group. Vastus medialis and vastus lateralis have been demonstrated to show different EMG behaviors in healthy elderly, ${ }^{32}$ possibly due to their differences in histochemical properties. ${ }^{33}$ Our findings suggest that quadriceps fatigability would be assessed with more sensitivity when EMG manifestations of fatigue are calculated from both vastus medialis and vastus lateralis rather than from only the vastus lateralis muscle.

\section{Limitations of the Study}

The main limitation of this study was the small sample size, which reduced the power of the results and the possibility of applying findings to the COPD population as a whole. Moreover, our sample was not balanced throughout the full spectrum of disease severity, since 8 of 14 subjects had a diagnosis of moderate COPD. Another important limitation was the absence of an age-matched control group. Our results on neuromuscular fatigue and exercise tolerance are likely to be influenced by age, making it difficult to interpret the results specifically for COPD subjects. The isometric contraction we used to assess fatigability was a task highly standardizable and easy to accomplish for the participants. However, such a task is far from real life movement and everyday physical demands. Future research should focus on overcoming the present limitations implementing the following advances: expanding the sample size to represent the full spectrum of disease severity, including an age-matched healthy control group, including the assessment of neuromuscular fatigue in dynamic contractions, and assessing the fatigability in other muscle 

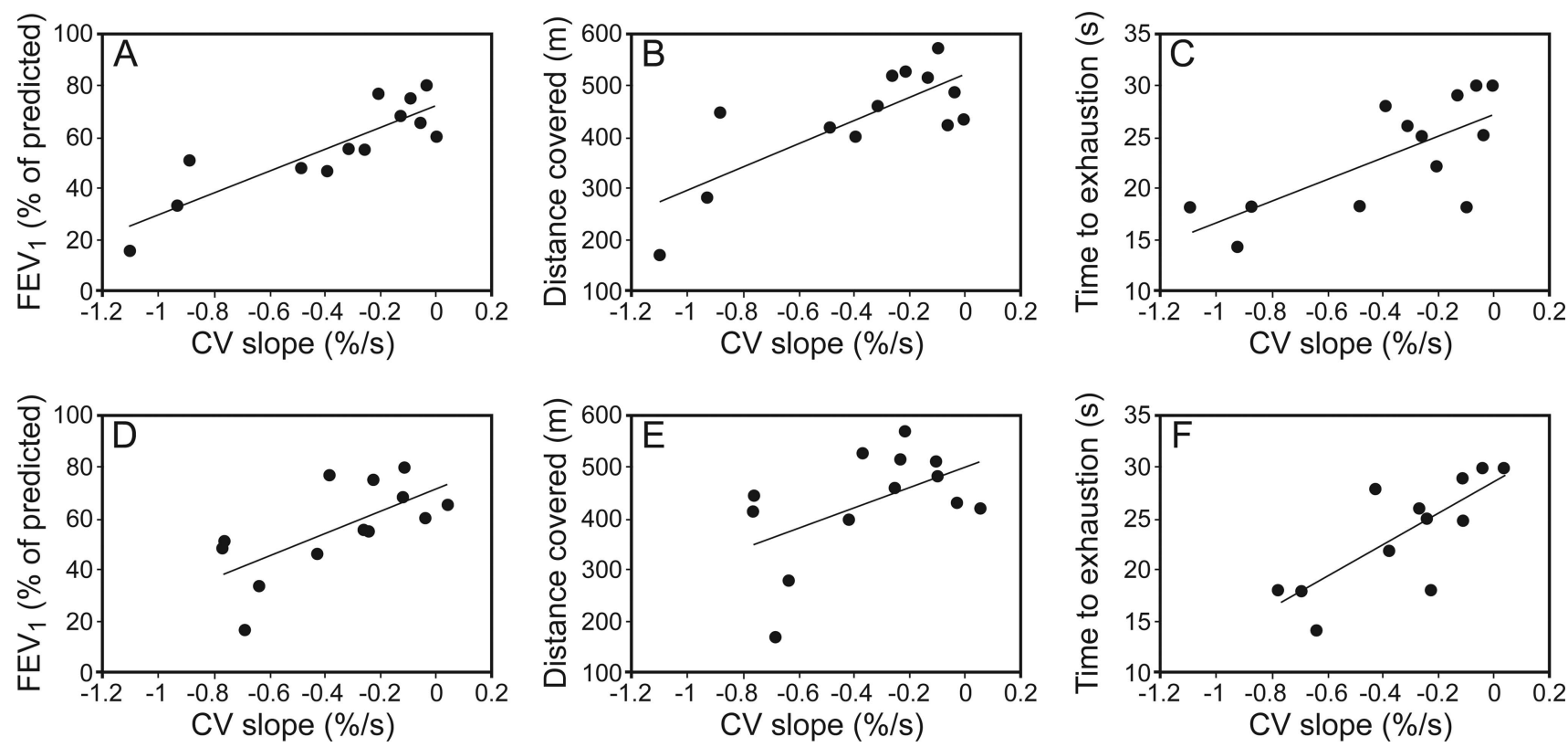

Fig. 2. Correlations between the rate of change of muscle fiber conduction velocity (CV) recorded on vastus lateralis (VL) and vastus medialis (VM) during the fatiguing contraction and pulmonary function $\left(\mathrm{FEV}_{1}\right)(\mathrm{A}$ and $\mathrm{D}), 6$-min walk test $(6 \mathrm{MWT})(\mathrm{B}$ and $\mathrm{E})$, and time to exhaustion $(\mathrm{C}$ and $\mathrm{F})$.

groups and with tasks more related to the activities of daily life.

\section{Conclusions}

First, the rate of change of conduction velocity during sustained quadriceps contraction significantly correlated with disease severity and exercise tolerance in subjects with mild to very severe COPD. Such correlations can be interpreted as evidence of increasing neuromuscular fatigability when disease stages increase. Second, the rate of change of conduction velocity calculated in the first $10 \mathrm{~s}$ of contraction was found to correlate with the time to exhaustion, disease severity, and exercise tolerance. This finding suggests that the conduction velocity rate of change could also be used to assess fatigability in non-exhausting contractions.

\section{ACKNOWLEDGMENTS}

We thank Valentina Bellini for valuable help with data collection.

\section{REFERENCES}

1. Decramer M, Janssens W, Miravitlles M. Chronic obstructive pulmonary disease. Lancet 2012;379(9823):1341-1351.

2. Vestbo J, Hurd SS, Agusti AG, Jones PW, Vogelmeier C, Anzueto A, et al. Global strategy for the diagnosis, management, and prevention of chronic obstructive pulmonary disease: GOLD executive summary. Am J Respir Crit Care Med 2013;187(4):347-365.

3. Gosker HR, Zeegers MP, Wouters EF, Schols AM. Muscle fibre type shifting in the vastus lateralis of patients with COPD is associated with disease severity: a systematic review and meta-analysis. Thorax 2007;62(11):944-949.

4. Kim HC, Mofarrahi M, Hussain SN. Skeletal muscle dysfunction in patients with chronic obstructive pulmonary disease. Int $\mathrm{J}$ Chron Obstruct Pulmon Dis 2008;3(4):637-658.

5. Barnes PJ, Celli BR. Systemic manifestations and comorbidities of COPD. Eur Respir J 2009;33(5):1165-1185.

6. Man WD, Kemp P, Moxham J, Polkey MI. Skeletal muscle dysfunction in COPD: clinical and laboratory observations. Clin Sci 2009;117(7):251-264.

7. González-Izal M, Malanda A, Gorostiaga E, Izquierdo M. Electromyographic models to assess muscle fatigue. J Electromyogr Kinesiol 2012;22(4):501-512.

8. Merletti R, Farina D, Gazzoni M. The linear electrode array: a useful tool with many applications. J Electromyogr Kinesiol 2003;13(1): 37-47.

9. Masuda K, Masuda T, Sadoyama T, Inaki M, Katsuta S. Changes in surface EMG parameters during static and dynamic fatiguing contractions. J Electromyogr Kinesiol 1999;9(1):39-46.

10. Merletti R, Knaflitz M, De Luca CJ. Myoelectric manifestations of fatigue in voluntary and electrically elicited contractions. J Appl Physiol 1990;69(5):1810-1820.

11. Blijham PJ, ter Laak HJ, Schelhaas HJ, van Engelen BG, Stegeman DF, Zwarts MJ. Relation between muscle fiber conduction velocity and fiber size in neuromuscular disorders. J Appl Physiol 2006; 100(6): 1837-1841.

12. Farina D, Merletti R, Enoka RM. The extraction of neural strategies from the surface EMG. J Appl Physiol 2004;96(4):1486-1495.

13. Rainoldi A, Galardi G, Maderna L, Comi G, Lo Conte L, Merletti R. Repeatability of surface EMG variables during voluntary isometric contractions of the biceps brachii muscle. J Electromyogr Kinesiol 1999;9(2):105-119.

14. Kupa EJ, Roy SH, Kandarian SC, De Luca CJ. Effects of muscle fiber type and size on EMG median frequency and conduction velocity. J Appl Physiol 1995;79(1):23-32. 
15. Sadoyama T, Masuda T, Miyata H, Katsuta S. Fibre conduction velocity and fibre composition in human vastus lateralis. Eur J Appl Physiol Occup Physiol 1988;57(6):767-771.

16. Merletti R, Rainoldi A, Farina D. Surface electromyography for noninvasive characterization of muscle. Exerc Sport Sci Rev 2001; 29(1):20-25.

17. Evans RA, Kaplovitch E, Beauchamp MK, Dolmage TE, Goldstein RS, Gillies CL, et al. Is quadriceps endurance reduced in COPD?: a systematic review. Chest 2015;147(3):673-684.

18. Merletti R, Roy S. Myoelectric and mechanical manifestations of muscle fatigue in voluntary contractions. J Orthop Sports Phys Ther 1996;24(6):342-353.

19. Harriss DJ, Atkinson G. Ethical standards in sport and exercise science research: 2014 update. Int J Sports Med 2013;34(12):1025-1028.

20. Miller MR, Hankinson J, Brusasco V, Burgos F, Casaburi R, Coates A, et al. Standardisation of spirometry. Eur Respir J 2005;26(2):319338.

21. Butland RJ, Pang J, Gross ER, Woodcock AA, Geddes DM. Two-, six-, and 12-minute walking tests in respiratory disease. Br Med J 1982;284(6329):1607-1608.

22. Hermens HJ, Freriks B, Disselhorst-Klug C, Rau G. Development of recommendations for SEMG sensors and sensor placement procedures. J Electromyogr Kinesiol 2000;10(5):361-374.

23. Mannion AF, Dumas GA, Stevenson JM, Cooper RG. The influence of muscle fiber size and type distribution on electromyographic measures of back muscle fatigability. Spine 1998;23(5):576-584.

24. Casale R, Rainoldi A, Nilsson J, Bellotti P. Can continuous physical training counteract aging effect on myoelectric fatigue? a surface electromyography study application. Arch Phys Med Rehabil 2003; 84(4):513-517.
25. Melchiorri G, Rainoldi A. Mechanical and myoelectric manifestations of fatigue in subjects with anorexia nervosa. J Electromyogr Kinesiol 2008;18(2):291-297.

26. Falla D, Rainoldi A, Merletti R, Jull G. Myoelectric manifestations of sternocleidomastoid and anterior scalene muscle fatigue in chronic neck pain patients. Clin Neurophysiol 2003;114(3):488-495.

27. Pedrinelli R, Marino L, Dell'Omo G, Siciliano G, Rossi B. Altered surface myoelectric signals in peripheral vascular disease: correlations with muscle fiber composition. Muscle Nerve 1998;21(2):201210.

28. Merletti R, Farina D, Gazzoni M, Schieroni MP. Effect of age on muscle functions investigated with surface electromyography. Muscle Nerve 2002;25(1):65-76.

29. Polkey MI, Hawkins P, Kyroussis D, Ellum SG, Sherwood R, Moxham J. Inspiratory pressure support prolongs exercise induced lactataemia in severe COPD. Thorax 2000;55(7):547-549.

30. Chavannes N, Vollenberg JJ, van Schayck CP, Wouters EF. Effects of physical activity in mild to moderate COPD: a systematic review. Br J Gen Pract 2002;52(480):574-578.

31. Allaire J, Maltais F, Doyon JF, Noël M, LeBlanc P, Carrier G, et al. Peripheral muscle endurance and the oxidative profile of the quadriceps in patients with COPD. Thorax 2004;59(8):673-678.

32. Boccia G, Dardanello D, Coratella G, Rinaldo N, Schena F, Rainoldi A. Differences in age-related fiber atrophy between vastii muscles of active subjects: a multichannel surface EMG study. Physiol Meas 2015;36(7):1591-1600.

33. Travnik L, Pernus F, Erzen I. Histochemical and morphometric characteristics of the normal human vastus medialis longus and vastus medialis obliquus muscles. J Anat 1995;187(2):403-411. 